\title{
Renormalization in Quantum Field Theory and the Riemann-Hilbert problem
}

\author{
Alain Connes* Dirk Kreimer ${ }^{\dagger}$ \\ Institut des Hautes Etudes Scientifiques \\ Le Bois Marie, 35 route de Chartres \\ F-91440 Bures-sur-Yvette, France \\ IHES/M/99/75, hep-th/9909126
}

\begin{abstract}
We show that renormalization in quantum field theory is a special instance of a general mathematical procedure of multiplicative extraction of finite values based on the Riemann-Hilbert problem. Given a loop $\gamma(z),|z|=1$ of elements of a complex Lie group $G$ the general procedure is given by evaluation of $\gamma_{+}(z)$ at $z=0$ after performing the Birkhoff decomposition $\gamma(z)=\gamma_{-}(z)^{-1} \gamma_{+}(z)$ where $\gamma_{ \pm}(z) \in G$ are loops holomorphic in the inner and outer domains of the Riemann sphere (with $\gamma_{-}(\infty)=1$ ). We show that, using dimensional regularization, the bare data in quantum field theory delivers a loop (where $z$ is now the deviation from 4 of the complex dimension) of elements of the decorated Butcher group (obtained using the Milnor-Moore theorem from the Kreimer Hopf algebra of renormalization) and that the above general procedure delivers the renormalized physical theory in the minimal substraction scheme.
\end{abstract}

\section{Introduction}

It has become increasingly clear in [1] [2] [3] that the nitty-gritty of the perturbative expansion in quantum field theory is hiding a beautiful underlying algebraic structure which does not meet the eye at first sight.

As is well known most of the terms in the perturbative expansion are given by divergent integrals which require renormalization. In [1] the renormalization technique was shown to give rise to a Hopf algebra whose antipode $S$ delivers the same terms as those involved in the subtraction procedure before the

*connes@ihes.fr

$\dagger$ kreimer@ihes.fr 
renormalization map $R$ is applied. In [2] the group $G$ associated to this Hopf algebra by the Milnor-Moore theorem was computed by exhibiting a basis and computing Lie brackets for its Lie algebra. It was shown that the collection of all bare amplitudes indexed by Feynman diagrams in dimensionally regularized perturbative quantum field theory is just a point $\phi$ in the group $G_{K}$, where $K=\mathbb{C}\left[z^{-1},[z]\right]$ is the field of Laurent series.

Though this made it clear that the Hopf algebra and its antipode are providing the correct framework to understand renormalization, some of the mystery was still around because of the somewhat ad hoc manner, in which the antipode $S$ had to be twisted by the renormalization map $R$ in order to fully account for the physical computations.

The twisted antipode $S_{R}$ is defined recursively [1, 3] by

$$
S_{R}(X)=-R\left[\phi(X)+S_{R}\left(X^{\prime}\right) \phi\left(X^{\prime \prime}\right)\right],
$$

where we abbreviate the coproduct of $X$ as $\Delta(X)=X \otimes 1+1 \otimes X+X^{\prime} \otimes X^{\prime \prime}$, omitting the summation sign for abbreviation always and assuming $\bar{e}(X)=0$, where $\bar{e}$ is the counit.

The introduction of the twisted antipode is imposed by the actual operations performed in renormalization. Thanks to multiplicativity constraints (m.c.s),

$$
R[X Y]-R[R[X] Y]-R[X R[Y]]+R[X] R[Y]=0,
$$

$S_{R}$ was shown in [3] to cover all algebraic aspects of multiplicative renormalization, $S_{R}(X Y)=S_{R}(Y) S_{R}(X)$, including scale transformations and changes of renormalization schemes by operations in the group.

But except from an obvious analogy between the recursive definition of $S_{R}$ and the usual recursive construction of the antipode $S$ the conceptual understanding of the twisted antipode was obviously missing.

We shall unveil the conceptual nature of $S_{R}$ thanks to the Riemann-Hilbert problem [5] for the group $G$.

This problem which together with the inverse scattering method has been a very successful tool to solve soliton equations, can be formulated as follows: For a given analytic curve $C \subset \mathbb{C} P^{1}$ and a map $\gamma$ from $C$ to a complex Lie group $G$, find the decomposition

$$
\gamma(z)=\gamma_{-}(z)^{-1} \gamma_{+}(z)
$$

of $\gamma$ as a pointwise product where $\gamma_{+}(z)$ (resp. $\gamma_{-}(z)$ ) is the boundary value of an holomorphic map from the inner (resp. outer) domain of $C$ to the group $G$ and $\gamma_{-}$is normalized by $\gamma_{-}(\infty)=1$. This decomposition is called the Birkhoff decomposition.

\footnotetext{
${ }^{1}$ In the simplest instance this group was later identified in 4 as the Butcher group of numerical analysis. For the general case we will use the terminology of decorated Butcher group.
} 
What we shall show is that the renormalized theory is obtained from the bare theory through the Riemann-Hilbert problem for the group $G$ as follows.

As we have seen above, given a quantum field theory, the collection of all bare amplitudes indexed by Feynman diagrams in dimensionally regularized perturbative quantum field theory is just a point $\phi$ in the group $G_{K}$, where $K=\mathbb{C}\left[z^{-1},[z]\right]$ is the field of Laurent series.

In fact, looking more closely, this bare data is encapsulated as a loop $\gamma(z)$ of elements in the group $G$, where $z$ is the complex deviation from dimension four and lies in a small circle $C$ around the origin.

The renormalized theory is just the evaluation at $D=4$ of the holomorphic piece $\gamma_{+}$in the Birkhoff decomposition of the loop $\gamma$ as a product of two holomorphic maps $\gamma_{ \pm}$from the respective connected components $C_{ \pm}$of the complement of the circle $C$ in the Riemann sphere $\mathbb{C} P^{1}$.

Thus, the nuance between naive subtraction of pole parts and renormalization by local counterterms is the same as passing from the additive Hilbert transform to the multiplicative Riemann-Hilbert problem.

This allows us to complete our understanding of the conceptual nature of renormalization and to assert that, contrary to its reputation, the subtraction procedure as applied by practitioners of QFT successfully over many decades is now backed by its conceptual significance.

\section{The twisted antipode and the Birkhoff decom- position}

Let $H$ be a positively graded Hopf algebra over $\mathbb{C}$ which is commutative as an algebra. Let $G$ be the group of characters of the algebra $H$, endowed with the group operation $\phi_{1} \star \phi_{2}$ given by the formula

$$
\phi_{1} \star \phi_{2}(X)=\phi_{1} \otimes \phi_{2}(\Delta(X)) \quad \forall X \in H
$$

where $\Delta$ is the coproduct.

We shall show in this section that the Riemann-Hilbert problem for $G$ yields exactly the defining equation for the twisted antipode $S_{R}$.

We let $\mathcal{R}$ be the ring of Laurent polynomials ${ }^{3}$ and $R: \mathcal{R} \rightarrow \mathcal{R}$ be the linear projection on the subalgebra $\mathcal{R}_{-}$generated by $z^{-1}$ parallel to the subalgebra $\mathcal{R}_{+}$generated by $1, z$.

Let us first recall for clarity the standard dictionary between the language of homomorphisms from $H \rightarrow \mathcal{R}$ (resp. to $\mathcal{R}_{ \pm}$) and the language of loops with values in $G$ and domain the inner and outer components $C_{ \pm}$of the complement

\footnotetext{
${ }^{2}$ We assume that the subspace $H_{n}$ of elements of degree $n$ is finite dimensional for each $n$ and that $H_{0}$ is reduced to scalars.

${ }^{3}$ The discussion also applies to the ring of meromorphic functions in $C_{+}$whose only singularity in $C_{+}$is a pole of finite order at 0 .
} 
of the unit circle $C$. We let $\tilde{H}$ be the augmentation ideal of $H$, ie. the kernel of the counit. The dictionary is then the following:

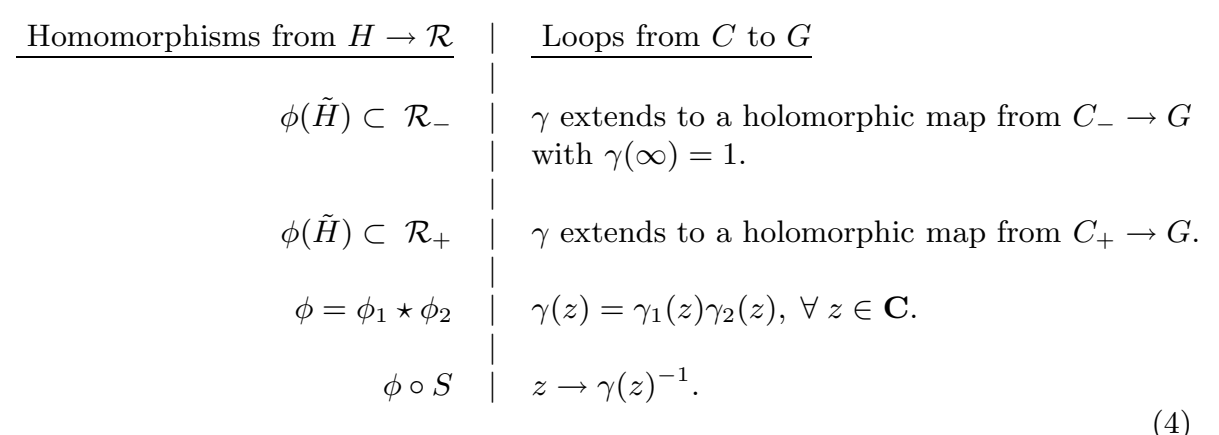

For elements $X \in \tilde{H}$ we shall use as above the short-hand notation

$$
\Delta(X)=X \otimes 1+1 \otimes X+X^{\prime} \otimes X^{\prime \prime}
$$

where we omit the summation and the components $X^{\prime}$ and $X^{\prime \prime}$ are of degree strictly less than that of $X$ for $X \in H_{n}, n>0$.

Theorem 1: Let $\phi$ be an homomorphism from $H \rightarrow \mathcal{R}$. The $\phi_{-}$-component of the Birkhoff decomposition $\phi=\phi_{-}^{-1} \star \phi_{+}$of the corresponding loop is characterized as the unique solution of the following equation

$$
\phi_{-}(X)=-R\left[\phi(X)+\phi_{-}\left(X^{\prime}\right) \phi\left(X^{\prime \prime}\right)\right], \forall X \in \tilde{H} .
$$

The main point of this theorem, of course, is that this equation is identical to the defining equation for the twisted antipode $S_{R}$.

Proof: By uniqueness of the solution of the Riemann-Hilbert problem it is enough to exhibit one solution. Let us define $\phi_{-}$by $\phi_{-}=\bar{e}$ (where $\bar{e}$ is the counit of $H$ ) on elements of $H$ of degree zero and then by induction, using the above equation. The first thing to check which is not obvious is that this definition actually yields a homomorphism. This follows from [3] using the multiplicativity property of the map $R$

$$
R[x y]-R[R[x] y]-R[x R[y]]+R[x] R[y]=0 .
$$

For the sake of completeness we briefly recall the main ingredients of the proof. It suffices to prove the assertion for homogeneous elements $X, Y$ of non-zero degree. This is done by induction on the sum of the respective degrees. Writing the recursive definition for both $\phi_{-}(X)$ and $\phi_{-}(Y)$, and taking the product yields, using (7) a sum of terms which one has to equate with the terms coming from the recursive definition applied to $X Y$. One has

$$
\begin{aligned}
\Delta(X Y)= & X Y \otimes 1+1 \otimes X Y+X \otimes Y+Y \otimes X+X^{\prime} Y \otimes X^{\prime \prime}+X^{\prime} \otimes X^{\prime \prime} Y \\
& +X Y^{\prime} \otimes Y^{\prime \prime}+Y^{\prime} \otimes X Y^{\prime \prime}+X^{\prime} Y^{\prime} \otimes X^{\prime \prime} Y^{\prime \prime} .
\end{aligned}
$$


Using the induction hypothesis, i.e. the multiplicativity of $\phi_{-}$together with the defining equation of $\phi_{-}$one easily reorganizes the above nine terms, using (6), to get the desired result.

Now that we know that $\phi_{-}$is a homomorphism we just need to check that $\phi_{-}(\tilde{H}) \subset \mathcal{R}_{-}$which is obvious by construction since $\mathcal{R}_{-}$is the range of the projection $R$, and that the product $\phi_{-} \star \phi$ lands in $\mathcal{R}_{+}$. But $\mathcal{R}_{+}$is the kernel of $R$ and it suffices to check that the composition $R\left[\phi_{-} \star \phi\right]$ vanishes. But this follows directly from the defining equation using $R\left[\phi_{-}\right]=\phi_{-}$.

\section{Multiplicative Renormalization}

Let us go back to the twisted antipode $S_{R}$. First of all, $S_{R}$ should be viewed as a homomorphism from the Hopf algebra $H$ to the ring of meromorphic functions in $C_{+}$whose only singularity in $C_{+}$is a pole of finite order at 0 . Its recursive definition starts with the given homomorphism $\phi$ from $H$ to $K$ and modifies it by induction using the above formula

$$
S_{R}(X)=-R\left[\phi(X)+S_{R}\left(X^{\prime}\right) \phi\left(X^{\prime \prime}\right)\right]
$$

while $S_{R}(e)=1$.

Theorem 2: The transition from the bare Green function $\phi(X)$ to the $M S$ renormalized Green function $\psi(X)=\left[S_{R} \star \phi(X)\right]$ is the multiplicative projection of the bare Green function $\phi$ to the holomorphic part $\phi_{+}$followed by evaluation at the origin. I

The proof of this theorem relies on the results of [1][2] 3], on theorem 1 and on the detailed discussion of dimensional regularization in the next section.

The relation between different renormalization schemes explained in [3] also explains the finiteness of Green functions when we vary dimensionful parameters. It is common practice to maintain dimensionless coupling constants in dimensional regularization by introducing a dimensionful parameter $\left[\mu^{2}\right]^{z}$ into the integral measure, as in the example exhibited in the next section. The Birkhoff decomposition is done at $q^{2}=\mu^{2}$, say. At other $q^{2}$, one imposes on Feynman graphs $\Gamma$ with $\operatorname{deg}(\Gamma)=n$ loops a homomorphism $\rho(\Gamma)=\left[\mu^{2} / q^{2}\right]^{z \operatorname{deg}(\Gamma)}$ which is in accordance with the m.c.s.

It is important to stress that in the above multiplicative renormalization procedure the precise location of the curve $\mathrm{C}$ is not crucial since one can move it freely by a homotopy in the complement of the singularities of the initial loop $\gamma$.

We shall end the paper with a few comments on the known crucial features of dimensional regularization which enabled us to obtain this result.

\footnotetext{
${ }^{4}$ This result is applicable as long as the theory under consideration allows for local counterterms, which includes renormalizable theories but also effective theories.
} 


\section{Dimensional regularization}

Dimensional regularization (Dim.Reg.) can be characterized by the three following properties: it naturally involves $\mathbb{C} P^{1}$, it projects to logarithmic divergences and it raises internal propagators to complex powers.

It promotes the dimension four of spacetime consistently to an analytic continuation to $D=4-2 z, z \in \mathbb{C}$, so that the complex parameter $z$ serves as a regularization parameter. It is not necessary to exhibit the technical details of this analytic continuation, we rather comment on its most useful properties mentioned above.

All the above three properties distinguish it from regularization prescriptions which use a dimensionful regularization parameter, like in a cut-off or PauliVillars scheme, where

- a dimensionful parameter $\lambda$ parametrizes the divergences by a finite series in $\lambda$ and $\log \lambda$, and the convergent part as a series in $1 / \lambda$, spoiling any attempt towards using $\lambda$ in a holomorphic decomposition of the finite and divergent part;

- it involves no projection onto logarithmic divergences at the level of the regularization;

- integration of subgraphs evaluates to logarithms of internal propagators instead of complex powers.

The practical advantages of Dim.Reg. in this respect are so severe that even a prescription like on-shell BPHZ, fully avoiding regularization at all, cannot compete with Dim.Reg. in practical calculations. We now see that this is underwritten from a conceptual viewpoint: it is actually the presence of complex domains provided by $z=(4-D) / 2 \in \mathbb{C}$ in Dim.Reg. which allows to promote renormalization to a concept.

Let us for the sake of the reader exhibit these features in some detail using simple examples. Let us consider the basic integral in dimensionally regularized Euclidean space

$$
\int \frac{\mathrm{d}^{D} k}{\left[\mu^{2}\right]^{-z}} \frac{1}{k^{2}+m^{2}}=-m^{2} \int \frac{\mathrm{d}^{D} k}{\left[\mu^{2}\right]^{-z}} \frac{1}{k^{2}\left(k^{2}+m^{2}\right)}+\int \frac{\mathrm{d}^{D} k}{\left[\mu^{2}\right]^{-z}} \frac{1}{k^{2}} .
$$

In Dim.Reg., the last expression on the rhs is zero thanks to analytic continuation, which gives [6], as one of Dim.Reg.s defining properties,

$$
\int \frac{\mathrm{d}^{D} k}{\left[\mu^{2}\right]^{-z}}\left[k^{2}\right]^{a}=0
$$

\footnotetext{
${ }^{5}$ See [7] for a typical example of a calculation which would have been barely possible without Dim.Reg. and the multiplicativity properties of counterterms.
} 
for all $a \in \mathbb{C}$. The first expression on the rhs of (5) evaluates to $-m^{2}\left[m^{2} / \mu^{2}\right]^{-z}$ $\pi^{2-z} \Gamma(-1+z)$, with the $\Gamma(-1+z)$-function delivering the expected pole term near $z=0$.

Evaluating the same quadratic divergent integrand by a standard integral in four dimension, multiplying the integrand with a cut-off function $\left.\Theta\left[\lambda-\sqrt{(} k^{2}\right)\right]$, we find the result $-m^{2} \pi^{2}\left[\log \lambda^{2} / m^{2}+\log \left(1+m^{2} / \lambda^{2}\right)\right]+\pi^{2} \lambda^{2}$, where $\lambda \rightarrow \infty$ is now the limit of interest.

What can we learn from this basic example? First of all, we use it to exhibit what we mean when we say that Dim.Reg. naturally involves $\mathbb{C} P^{1}$. Clearly, the bare result in Dim.Reg. is a series which has a pole in $z$ of finite order (first order in the example) and is an infinite Taylor series in $z$. We hence can formulate the quest for the Birkhoff decomposition in the framework used above.

Further, the result $\int \frac{\mathrm{d}^{D} k}{\left[\mu^{2}\right]^{-z}}\left[k^{2}\right]^{a}=0$ immediately ensures that one only confronts logarithmic divergent integrals, with suitable dimensionful coefficients $\left(-m^{2}\right.$ in our example) maintaining the correctness of powercounting. This has far reaching consequences: it allows to add zero in a suitable manner to each Feynman integrand to dispense with all appearances of overlapping divergences, as exemplified by the following instructive example:

$$
\int \frac{d^{D} k}{\left[\mu^{2}\right]^{-z}} \frac{d^{D} l}{\left[\mu^{2}\right]^{-z}} \frac{1}{\left(k^{2}+m^{2}\right)(k+l)^{2} l^{2}}=\int \frac{d^{D} k}{\left[\mu^{2}\right]^{-z}} \frac{d^{D} l}{\left[\mu^{2}\right]^{-z}}\left[\frac{-m^{2}}{\left(k^{2}+m^{2}\right) k^{2}(k+l)^{2} l^{2}}\right],
$$

where the lhs and rhs are equal using an addition of

$$
0=-\iint \frac{d^{D} k}{\left[\mu^{2}\right]^{-z}} \frac{d^{D} l}{\left[\mu^{2}\right]^{-z}} \frac{1}{k^{2}(k+l)^{2} l^{2}} .
$$

In a regularization using a dimensionful parameter, such a simplification would not appear until one uses the Hopf algebra structure to realize that terms which do not depend on dimensionful parameters drop out at the end of the calculation in ratios of the form $S_{R} \star \phi$. Hence, in Dim.Reg. we already obtain at the level of bare diagrams a decomposition of amplitudes into functions representing rooted trees, hence the desired representation in terms of elements of the decorated Butcher group $G_{K}$.

\section{Acknowledgements}

Both of us are grateful to the Clay Mathematics Institute and to Institut des Hautes Etudes Scientifiques for making our collaboration possible. D.K. thanks the DFG for a Heisenberg Fellowship.

\section{References}

[1] D. Kreimer, Adv.Theor.Math.Phys.2 (1998) 303, q-alg/9707029. 
[2] A. Connes, D. Kreimer, Commun.Math.Phys.199 (1998) 203, hepth/9808042.

[3] D. Kreimer, Adv.Theor.Math.Phys.3.3 (1999) hep-th/9901099.

[4] C. Brouder, Runge-Kutta methods and renormalization, hep-th/9904014.

[5] Mathématique et Physique, (Séminaire de l'ENS 79-82). Progress in Math. 37, Birkhäuser, Boston (1983).

[6] J.C. Collins, Renormalization, Cambridge Univ.Press 1984.

[7] D.J. Broadhurst, Four loop Dyson-Schwinger-Johnson anatomy, to appear in Phys.Lett.B, hep-ph/9909336. 